Evaluation of a medication calculation mobile app using a cognitive load instructional design

\author{
McMullan, Miriam
}

http://hdl.handle.net/10026.1/11998

10.1016/j.jimedinf.2018.07.005

International Journal of Medical Informatics

Elsevier

All content in PEARL is protected by copyright law. Author manuscripts are made available in accordance with publisher policies. Please cite only the published version using the details provided on the item record or document. In the absence of an open licence (e.g. Creative Commons), permissions for further reuse of content should be sought from the publisher or author. 
Evaluation of a medication calculation mobile app using a cognitive load instructional design

Miriam McMullan PhD

Lecturer

School of Health Professions

University of Plymouth

Peninsula Allied Health Centre

Derriford Road

Plymouth PL6 8BH

UK

Tel.: +44 1752588829

e-mail: mmcmullan@plymouth.ac.uk 


\title{
Evaluation of a medication calculation mobile app using a cognitive load instructional design
}

\begin{abstract}
Objective: For students attending healthcare courses, such as nursing and paramedicine, the ability to calculate medication dosages accurately is an essential but often a complex cognitive skill to be learned. This study aimed to determine whether a 'mastering medication calculation' mobile app, developed using an instructional design based on cognitive load theory, could improve students' medication calculation ability and self-efficacy. Additional objectives were: (1) To examine the correlations between students' medication calculation ability, self-efficacy and their numerical ability; (2) To explore how well medication calculation ability can be predicted by students' numerical ability and their medication calculation self-efficacy; (3) To obtain students' opinions regarding the mobile app's usefulness and usability.
\end{abstract}

Method: A quasi-experimental study was carried out with a convenience sample of 60 second year UK university healthcare students who used the app for 8 weeks while in clinical practice. Students were given medication calculation ability and self-efficacy tests pre- and post-intervention. In addition, they were asked pre-intervention to complete a numeracy test and to complete a post-intervention survey to obtain their opinions on the usefulness of the app.

Results: Following the use of the app students were significantly more able $(p=0.004)$ and more confident $(p<0.001)$ in performing medication calculations. There were significant positive correlations found between students' numerical ability and 
medication calculation ability $(p<0.001)$ and between students' medication calculation ability and self-efficacy $(p<0.001)$. Hierarchical multiple regression analysis indicated numerical ability $(\beta=0.46, p<0.001)$ and medication calculation self-efficacy $(\beta=0.46$, $p<0.001)$ to be the strongest predictors of medication calculation ability, with age and gender being non-significant. All students indicated the app to be an excellent method to support them in learning how to perform medication calculations.

Conclusion: This study indicated that the app's instructional design, based on cognitive load theory, significantly improved students' medication calculation ability and selfefficacy. Although the app's usability was highly rated by the students, further research is needed to determine if the app's instructional design can be used by learners of different levels of expertise and whether the design can be adopted for the learning of other complex cognitive skills. As numerical ability is essential for medication calculation ability, early identification and rectification of any numerical deficits is recommended.

Keywords: Cognitive load theory; Instructional design; Mobile learning; Medication calculations; Self-efficacy 


\section{Introduction}

Many healthcare students struggle with performing medication calculations [1]. A survey with 2,413 UK medical students and newly qualified doctors found that only $24 \%$ felt confident in performing medication calculations. The majority of the respondents $(74 \%)$ felt that the teaching and training of medication calculations had been inadequate and welcomed any additional educational interventions that could improve their competency [2]. One potential way of supporting students developing their medication calculation skills is by using mobile learning. It enables learning to take place anytime, anywhere and, for healthcare students, it enables students to practice and reinforce their calculation skills while being in clinical settings, thereby linking theory to practice [3]. To support students cultivating this essential skill, an interactive medication calculation app was developed based on cognitive load theory, which is an important instructional theory for learning complex, cognitive tasks $[4,5]$.

Cognitive load theory is concerned with the processing and storage of information in our memories [5]. Knowledge and/or skills are stored in the long-term memory in the shape of hierarchical information networks known as 'schemas' [6]. However, before these schemas can be constructed the information has to be processed by the working memory which has limited capacity, as it can only deal with about 4-6 elements of new information simultaneously [7] (Fig. 1). 


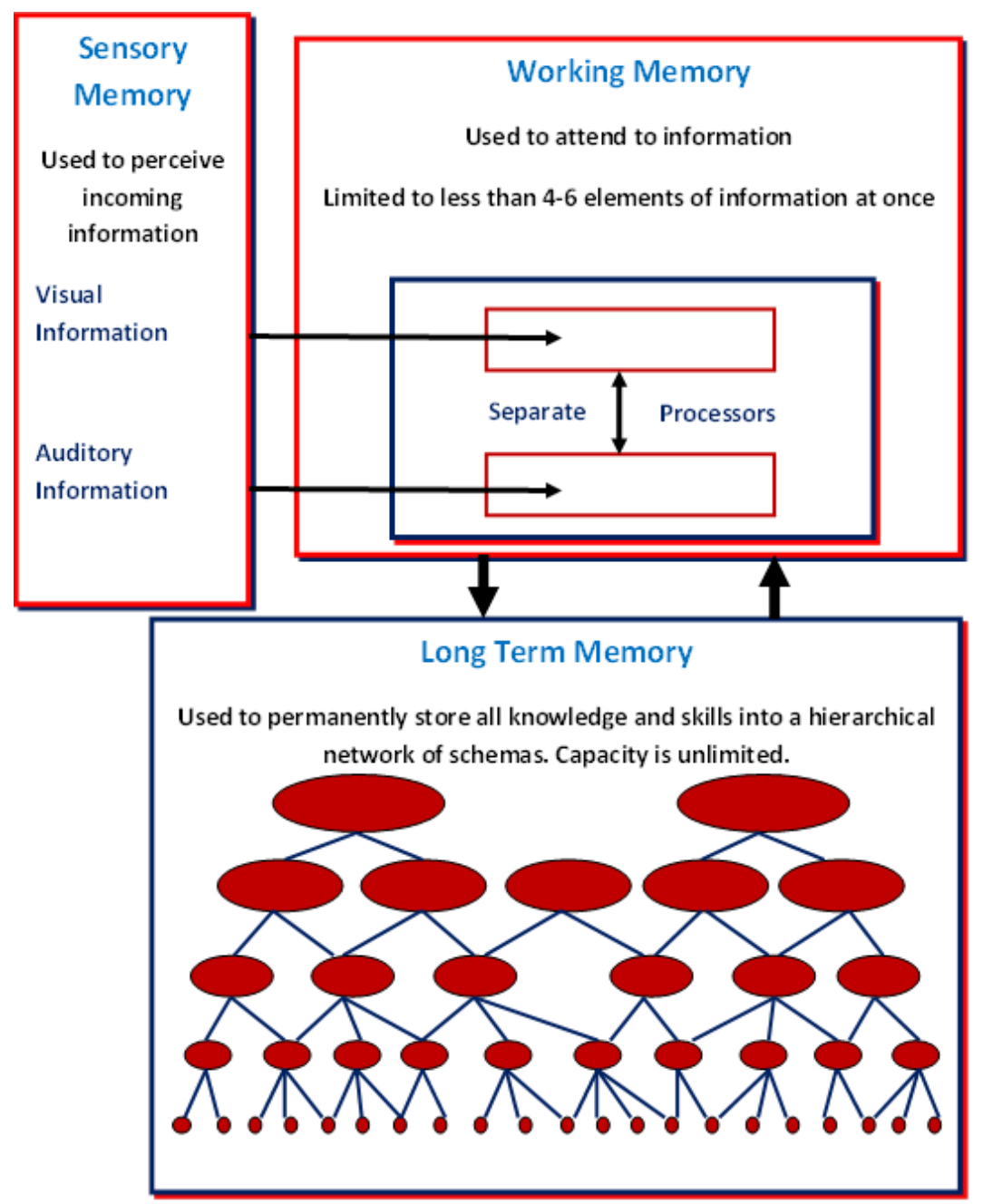

Fig. 1: Information processing model

The cognitive load acting on the working memory consists of two main types, namely intrinsic and extraneous [5]. Intrinsic cognitive load is the load placed on the working memory by the intrinsic nature of the materials to be learned with the magnitude of the intrinsic load depending on the learner's prior level of domain expertise i.e. schema development [8]. Extraneous load, on the other hand, is the load placed on the working memory due to the instructional design used to present the information. Badly designed learning material and instruction will increase the load on the working memory and 
thereby will reduce the working memory resources available for learning [9]. The loads are additive and, if learning is to occur, the total combined load must not exceed the working memory capacity [10].

\subsection{Application of Cognitive Load Theory to Instructional Teaching Design}

Cognitive load theory has broad implications for instructional design. If the instructional designer can reduce the intrinsic and/or extraneous load, learning can be increased without overloading the working memory [11]. The instructional technique that is particularly interesting for reducing the extraneous load is the worked example. Worked examples show learners, step-by-step, how to solve different problems and, for novice learners, impose a low cognitive load [12]. They essentially allow learners to be free from performance demands so that they can concentrate on gaining understanding [8].

Although worked examples are important in the early stages of skill acquisition, in later stages the actual problem solving is more beneficial for students' learning [8]. Therefore, with respect to the instructional design, to accommodate the learner's increase in expertise during practice, worked examples should gradually be replaced by conventional problem solving. This instructional strategy of 'fading out' the worked examples, while the learner's expertise increases, is known as the 'guidance fading strategy' [13]. 


\section{Medication Calculation App Development}

The overall development of the 'mastering medication calculation' mobile app

(subsequently referred to as mobile app or app) took a year. This consisted of an initial exploratory survey with 56 paramedic and operating department practice (ODP) students. Findings were that the most desirably features students would like to see in the app were interactivity, worked out examples, followed by tests and exercises. The app's content was developed with the aid of textbooks and academic experts in medication calculations who screened and reviewed the questions and scenarios. The content was subsequently transferred into a mobile application by a computing expert. For the app to work on both Android and iOS devices it was important to develop a fluid interface that would cope with the multitude of resolutions whilst retaining a usable and intuitive, interactive interface. Following iterative prototype testing by the students of the app's usability, leading to improved interface and interactivity, the app was evaluated (by a different cohort of students) on its effectiveness in improving students' medication calculation ability and self-efficacy.

As the app was aimed to be ultimately used by both novice and experienced health professionals, it was important that the content would be at the appropriate level and would reflect real-life scenarios. It was therefore decided that the most important medication calculations topics to be covered in this app had to be the basic calculations of solid (tablets) and liquid (injections) medications, as well as the more complex calculations of percentage solutions, drip and infusion rates. To incorporate the 'guidance fading strategy' design into the app [13], each topic started with two or three 
worked examples, which were followed by four or five problem solving exercises with linked solutions. Finally, to finish each topic, when learners felt confident and competent, the learners could self-test their understanding of that particular topic by completing a conventional, interactive problem-solving mini-quiz consisting of four to six questions. See Fig. 2 for examples of app screenshots.
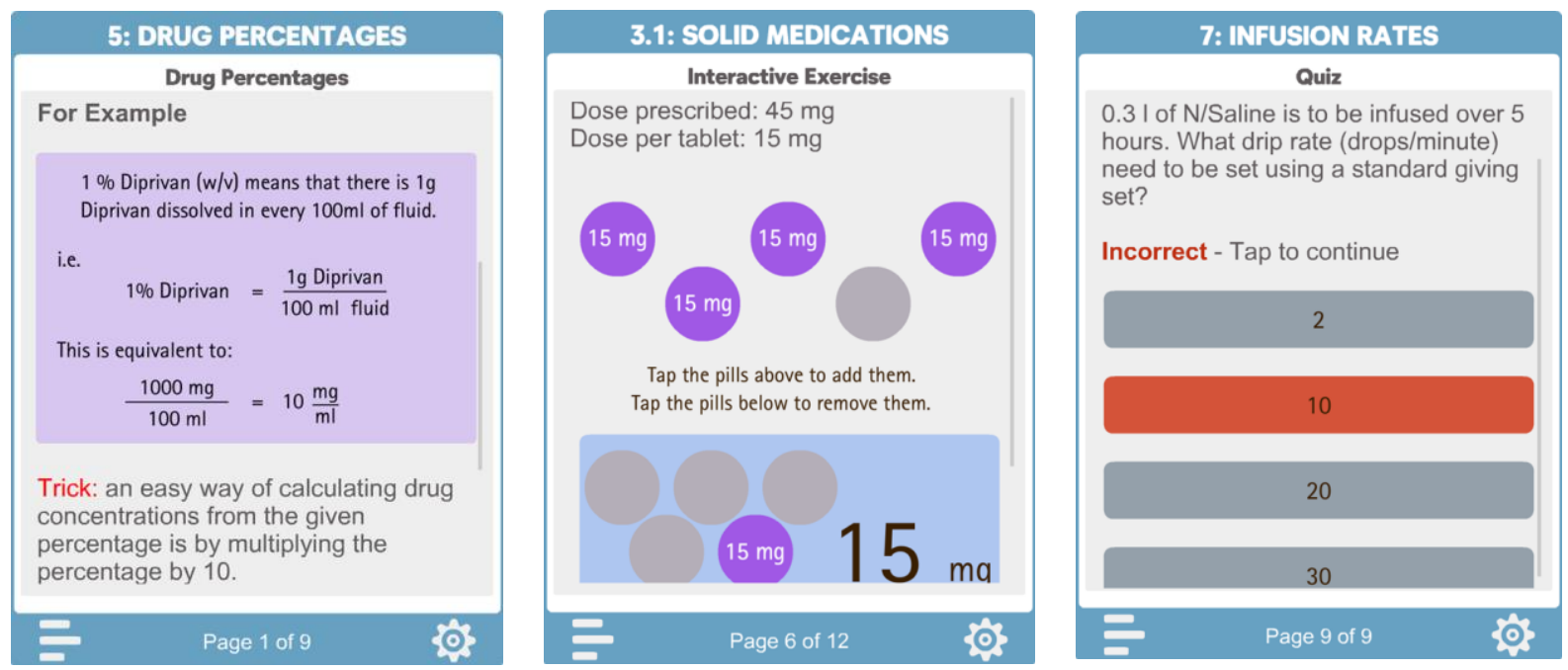

Fig. 2: Mobile app screenshots of a worked example, interactive exercise and quiz

\section{Methods: Medication Calculation App Evaluation}

The main aim of the present study was to determine whether a medication calculations mobile app design, based on cognitive load theory, improved students' medication calculation ability and confidence (self-efficacy) in performing medication calculations. Additional objectives of the study were: (1) To examine the correlations between students' medication calculation ability, self-efficacy and their numerical ability; (2) To explore how well medication calculation ability can be predicted by students' numerical 
ability and their self-efficacy in performing medication calculations (3) To obtain students' opinions regarding the mobile app's usefulness and usability.

Approval by the university research human ethics committee (12/13-63) was obtained before study commencement. All students were given an information letter, during one of their lectures, containing contact details in case students required further information before deciding to participate or if they wanted to be kept informed of the results of the study. Participation was voluntary and anonymity of data was guaranteed. For the tests, the demographic data obtained were students' registration number, program of study, age and gender. The survey was completely anonymous. Return of the tests and survey implied informed consent.

To evaluate the effectiveness of the mobile app in improving healthcare students' medication ability and self-efficacy, a one-group pre-test post-test quasi-experimental study was carried out with a convenience sample of all the second year paramedicine $(n=41)$ and ODP students $(n=19)$ studying at a UK university who used the app for 8 weeks while on clinical placement. The study was quasi-experimental due to the lack of a control group. Of the students, $55 \%$ were female and $60 \%$ were 24 years or younger, with $3 \%$ of the students aged over 45 .

To determine students' medication calculation ability and self-efficacy, students were given pre- and post-intervention: (i) a 20-item non-calculator medication calculation test. To avoid students having remembered the pre-intervention calculation answers, 
different numbers were used for the post-intervention calculation questions. The tests covered the main types of medication calculations paramedic and ODP healthcare professionals needed to know i.e. calculating dosages for liquid, oral and solid medications, injections, percentage solutions and intravenous fluids; (ii) A six item, 10point VAS scales $(0=$ lowest, $10=$ highest), medication calculation self-efficacy test for students to self-rate their confidence in performing medication calculations. A pilot study with 20 first year ODP students found a good internal reliability of the scale with a Cronbach's alpha of 0.93 .

To determine students' numerical ability, students were given pre-intervention a 15-item non-calculator validated basic numeracy test covering key calculation skills such as multiplication, fractions, percentages, decimals, conversions [14]. The face and content validity of these tests had been independently obtained from three healthcare academics with expertise in numerical and medication calculations.

Finally, to obtain students' opinions of the usefulness of the app, students completed a post-intervention paper-based survey. It consisted of a 10 item (see Table 1), 5-point Likert statements ( $1=$ strongly disagree to $5=$ strongly agree) scale, based on a validated scale reporting a Cronbach's alpha of 0.93 [15]. The internal consistency (reliability) of the scale used in this study's survey was good (Cronbach's alpha $=0.83$ ). The survey also contained an open question to enable students to provide more detailed, qualitative information. 
Table 1: Survey items obtaining students' opinions on usefulness of mobile app

1. The app will enhance my learning of drug calculations.

2. I will be able to use what I learned.

3. The app is an effective way to support my learning of drug calculations.

4. The activities give me practice and feedback.

5. The layout of the material is appropriate.

6. I like the interactivity of the app.

7. The app is easy to navigate.

8. The app is accessible.

9. The app is user friendly.

10. I enjoy learning about drug calculations this way.

\subsection{Data analysis}

Both descriptive and inferential statistical analyses were applied to the quantitative data using IBM SPSS Statistics V22.0. To determine if there were any differences pre- and post-intervention paired samples t-tests were performed. Pearson product-moment correlation analysis was used to examine relationships between variables. Hierarchical multiple regression analysis was applied to determine any statistically significant explanatory variables. Any qualitative data was analysed thematically using NVivo 10.

\section{Results}

The mean score students obtained for the pre-intervention numerical ability test was $58.7 \%(\mathrm{SD}=19.8)$. To determine if there were differences pre- and post-intervention in medication calculation ability and self-efficacy, paired-samples t-tests indicated a 
significant statistical improvement post-intervention in students' medication calculation ability $(\mathrm{t}=3.29, \mathrm{p}=0.004)$ and self-efficacy $(\mathrm{t}=6.27, \mathrm{p}<0.001)$ (Table 2$)$.

Table 2: Numerical Ability, Medication Calculation Ability and Self-Efficacy Pre-Post Intervention

\begin{tabular}{lccccc}
\hline & Pre-Intervention & & Post-Intervention & & t-test \\
\cline { 2 - 2 } & Mean (SD) \% & & Mean (SD) \% & & $p$ \\
\hline Drug Calculation Ability & $47.6(23.4)$ & & $56.7(24.7)$ & & 0.004 \\
Drug Calculation Self-Efficacy & $20.4(18.0)$ & & $49.6(19.9)$ & & $<0.001$ \\
Numerical Ability & $58.7(19.8)$ & & & \\
\hline
\end{tabular}

Significant positive correlations were found between students' performance in the medication calculation ability test and numerical ability test $(r=0.48, p<0.001)$ and between students' medication calculation ability and their confidence in performing medication calculations $(r=0.46, p<0.001)$.

\subsection{Medication Calculation Ability Prediction}

Prior to performing hierarchical multiple regression analysis, homoscedasticity, multicollinearity, normality and linearity between dependent (medication calculation ability) and independent variables (numerical ability, medication calculation self-efficacy, age and gender) were checked. Collinearity diagnostics showed the VIF and tolerance values to be respectively 1.0 and 0.9 , which indicated there was no multicollinearity present. In addition, all the points in the normal probability plot of the regression standardised residuals were lying in a reasonably straight diagonal line from bottom left 
to top right which showed there was no major deviation from normality. Results indicated the overall relationship to be significant $(F[4,52]=10.02, p<0.001)$ with $43.5 \%$ of the variance explained by our model $\left(R^{2}=43.5 \%\right)$. After controlling for the effects of age and gender, numerical ability and self-efficacy explained $42.3 \%$ of the variance in medication calculation ability. Both numerical ability $(\beta=0.46, p<0.001)$ and medication calculation self-efficacy $(\beta=0.46, p<0.001)$ made a unique statistically significant contribution. Neither age nor gender made a significant contribution (Table $3)$.

Table 3: Summary of Hierarchical Regression Analysis for Variables predicting medication calculation ability

\begin{tabular}{|c|c|c|c|c|c|c|c|c|}
\hline \multirow[b]{2}{*}{ Variable } & \multicolumn{4}{|c|}{ Model 1} & \multicolumn{4}{|c|}{ Model 2} \\
\hline & $B$ & SE B & $\beta$ & $p$ & $B$ & SE B & $\beta$ & $p$ \\
\hline Age & -1.90 & 2.65 & -0.10 & 0.48 & -2.29 & 2.04 & -0.12 & 0.27 \\
\hline Gender & -1.36 & 4.38 & -0.04 & 0.76 & 4.11 & 3.51 & 0.13 & 0.25 \\
\hline Numerical Ability & & & & & 0.37 & 0.90 & 0.46 & $<0.001$ \\
\hline $\begin{array}{l}\text { Medication Calculation } \\
\text { Self-Efficacy }\end{array}$ & & & & & 0.36 & 0.90 & 0.46 & $<0.001$ \\
\hline $\mathrm{R}^{2}$ & & 0.01 & & 0.72 & & 0.43 & & $<0.001$ \\
\hline
\end{tabular}

\subsection{Survey Findings}

All the students felt that the interactive mobile app was an excellent method to support them in learning how to perform medication calculations. Reasons ranged from the app providing 'learning enjoyment' (60\%) to 'effective way to learn' / 'user friendly' (91\%) (Fig. 3). 


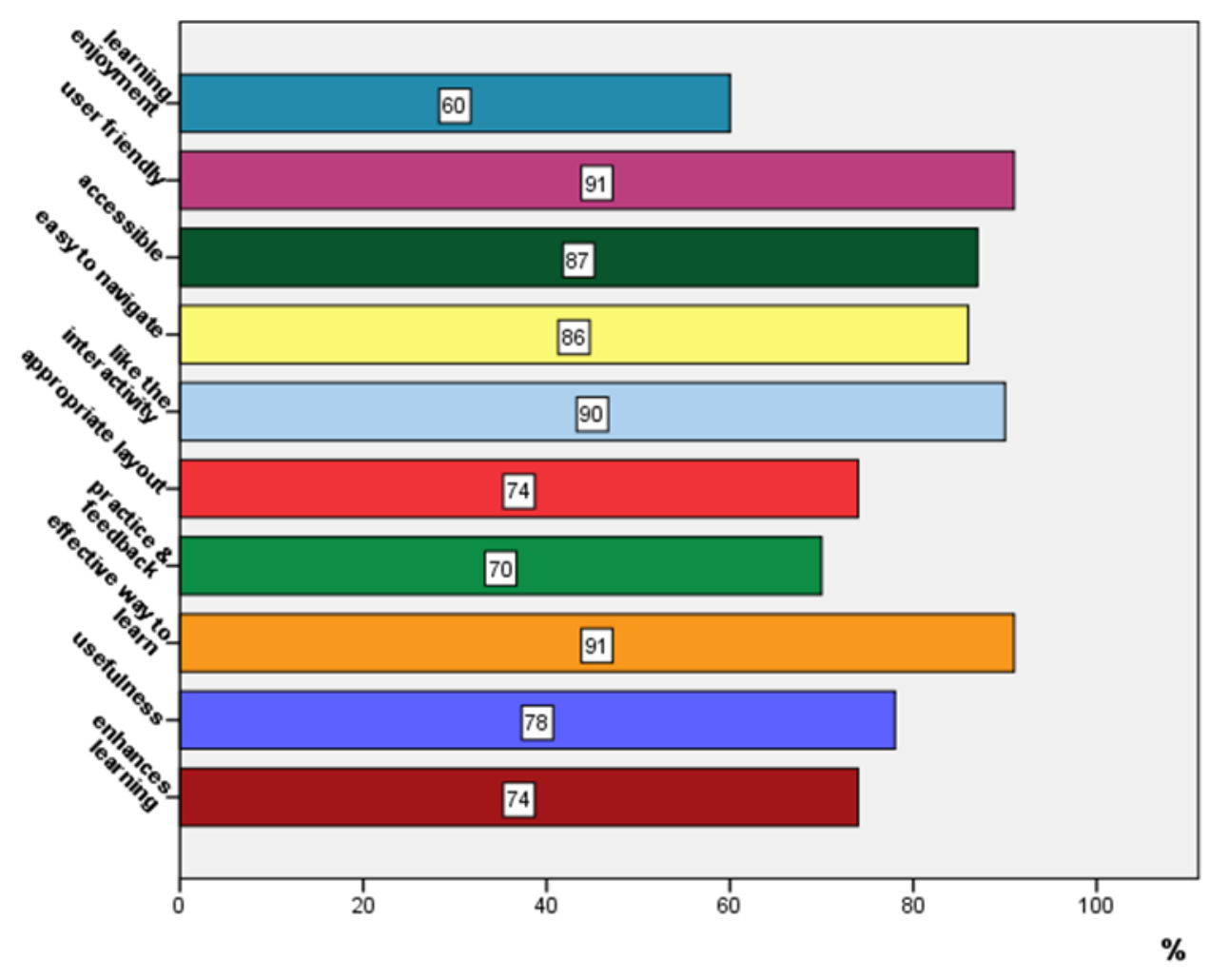

Fig. 3: Percentage of participants who agreed/strongly agreed with the medication calculations app statements

After thematic analysis of the students' answers regarding the app's usefulness and usability, three main themes emerged:

(i) Mobile App Instructional Design

- "It [the app] provided good step-by-step explanations, was easily understood and the examples and quizzes helped to practise and reaffirm knowledge gained thereby increasing my confidence."

- "The app is easy to use, clear, concise and logically laid out"

(ii) Relevance for future practice 
- "Easy reference tool and an extremely useful revision tool for when I am qualified."

- "I really enjoyed using this app. It will enhance my clinical practice"

(ii) Advantages of mobile learning

- "I always use my phone as a resource and the app allows me to practice anywhere, anytime."

- "It [the app] would provide a quick reference guide to work out drug calculations for our patients in an out-of-hospital high-pressured environment."

\section{Discussion}

The study results indicated that for learning a complex cognitive skill, such as medication calculations, using a mobile app whose instructional design is based on cognitive load theory significantly improves students' confidence and competence in performing that skill. Findings from the survey also indicated that students liked the app's instructional design. They all indicated that the mobile app was an excellent method to support them with their medication calculations, not just for while they were students but also for when they were qualified. They particularly liked the app's interactivity, its ease of use and accessibility.

The app in this study was designed to generate learning and understanding by reducing the cognitive load on the working memory. It did this by reducing the extraneous load through the use of worked examples and through the use of the 'guidance fading' strategy which fades out the use of worked examples to using problem solving 
exercises [9]. This approach confirmed results obtained by previous studies which demonstrated that using worked examples for novices' learning is very effective [16]. However, they also showed that when the learner becomes more expert at performing a skill these worked examples become redundant [12]. At this point, the major goal to be achieved is to increase the speed and accuracy of the performance and for the skill to become automated, which can only be achieved by the students practicing problem solving as opposed to only studying examples [8]. The effectiveness of 'fading out' worked examples was also confirmed by a study in the area of probability [17] which demonstrated that using a 'guidance fading' strategy led to better learning outcomes than having no fading present.

Apart from this study demonstrating a significant improvement in students' medication calculation ability and self-efficacy, results also indicated a strongly significant relationship between students' pre-intervention medication calculation ability and their numerical ability. This result, together with the regression analysis indicating numerical ability to be a main predictor of medication calculation ability, demonstrates the importance of students' numerical ability for performing medication calculations. Previous studies have indicated that many healthcare students struggle to perform basic numerical calculations [1,18], which was also the case in this study. The reasons for this poor performance can only be speculated upon, but could include dependency on using calculators [18]. 


\section{Conclusion and recommendations}

The medication calculations app instructional design based on cognitive load theory proved to be very successful as students in this study were significantly more able and confident in performing medication calculations following the use of the app. However, a limitation of the study was the lack of a control group. To overcome this, future research could compare the use of the app versus other learning materials using a randomised controlled trial design. Although the app had also been designed to be used by learners with different levels of expertise, i.e. from novice learners who need to work through the material from start to finish to more experienced learners who might only want to check and refresh their understanding of a particular topic, this was not evaluated in this study. It is therefore recommended that further research is carried out to determine whether this app's instructional design can be used by learners of different levels of expertise and also if the design can be adopted for the learning of other complex cognitive skills. It would also be interesting for future studies to investigate in more detail into how the app was used by the students, such as frequency and duration of app use; which were the most accessed topics. Finally, as numerical ability is essential for medication calculation ability and shown to be poor in this study, early identification and rectification of students' numerical deficits is recommended.

\section{Acknowledgements}

This work was supported by an individual HEA Teaching Development Grant.

\section{Conflicts of interest}

Declarations of interest: None 


\section{Summary Points}

What was already known on the topic:

- Calculating medication dosages accurately is an essential but often a complex cognitive skill to be learned.

- Cognitive load theory is an important instructional theory for learning complex, cognitive tasks.

- A potential method to support students to develop their medication calculation skills, particularly while they are in clinical practice, is by using mobile learning.

What this study added to our knowledge:

- The medication calculation mobile app's instructional design, based on cognitive load theory, significantly improved students' medication calculation ability and self-efficacy.

- Students greatly valued the mobile app's interactive instructional design as it provided clear explanations, provided practice and helped them to consolidate knowledge.

- As numerical ability is essential for performing medication calculations, any numerical deficits need to be identified and rectified as soon as possible. 


\section{References}

[1] S. Conroy, W.D Carroll, Prescribing in paediatrics, Archives of Disease in Childhood Education and Practice Edition 200994 (2009) 55-59.

[2] A. Heaton, D.J. Webb, S.R.J. Maxwell, Undergraduate preparation for prescribing: the view of 2,413 UK medical students and recent graduates, British Journal of Pharmacology 66 (2008) 128-134.

[3] H. Gatzke, J.E. Ransom, New skills for a new age: Preparing nurses for the $21^{\text {st }}$ century, Nursing Forum 36 (3) (2001) 13-17.

[4] F. Paas, J. Sweller, Implications of cognitive load theory for multimedia learning, in:

R.E. Mayer (Ed.), The Cambridge handbook of multimedia learning, Cambridge University Press, New York, 2014, pp. 27-42.

[5] J. Sweller, P. Ayres, S. Kalyuga, Cognitive load theory, Springer, New York, 2011.

[6] J. Van Merrienboer, J. Sweller, Cognitive load theory in health professional education: design principles and strategies, Medical Education 44 (1) (2010) 85-93.

[7] N. Cowan, Working Memory Capacity (Essays in Cognitive Psychology), Psychology Press, New York, 2005.

[8] A. Renkl, R.K. Atkinson, Structuring the Transition From Example Study to Problem Solving in Cognitive Skill Acquisition: A Cognitive Load Perspective, Educational Psychologist 38 (1) (2003) 15-22.

[9] J. Van Merrienboer, L. Kester, F. Paas, Teaching Complex Rather Than Simple Tasks: Balancing Intrinsic and Germane Load to Enhance Transfer of Learning, Applied Cognitive Psychology 20 (2006) 343-352. 
[10] F. Paas, J.E. Tuovinen, H. Tabbers, P.W.M. Gerven, Cognitive load measurement as a means to advance cognitive load theory, Educational Psychologist 38 (2003) 6371.

[11] O. Chen, G. Woolcott, J. Sweller, Using cognitive load theory to structure computerbased learning including MOOCs, Journal of Computer Assisted Learning 33 (2017) 293-305.

[12] P.A. Kirschner, J. Sweller, R.E. Clark, Why Minimal Guidance During Instruction Does Not Work: An Analysis of the Failure of Constructivist, Discovery, Problem-Based, Experiential, and Inquiry-Based Teaching, Educational Psychologist 41 (2) (2006) 7586.

[13] A. Renkl, Toward an instructionally oriented theory of example-based learning, Cognitive Science 38 (2014) 1-37.

[14] M. McMullan, R. Jones, S. Lea, Math anxiety, self-efficacy and ability in British undergraduate nursing students, Research in Nursing \& Health 35 (2012) 178-186. [15] M. McMullan, R. Jones, S. Lea, The effect of an interactive e-drug calculations package on nursing students' drug calculation ability and self-efficacy, International Journal of Medical Informatics 80 (2011) 421-430.

[16] R.K. Atkinson, S.J. Derry, A. Renkl, D. Wortham, Learning from examples: Instructional principles from the worked examples research, Review of Educational Research 70 (2000) 181-214.

[17] A. Renkl, R.K. Atkinson, C.S. Grose, How fading worked solution steps works: A cognitive load perspective, Instructional Science 32 (1-2) (2004) 59-82. 
[18] M. McMullan, R. Jones, S. Lea, Patient safety: Numerical skills and drug calculation abilities of nursing students and Registered Nurses, Journal of Advanced Nursing 66 (4) (2010) 891-899. 